January 1990 to 30 June 2006, based on linked records of the use of mental health services, hospital admissions, Medicare claims for GP and specialist services, electoral roll registration and deaths. Adjusted rate ratios (ARRs) for the number of visits to GPs by $\mathrm{MHCs}$ relative to non-MHCs, and for different categories of mental disorders.

Results Relative to non-MHCS, the ARR of visits to GPs by MHCs was 1.622 (95\% CI 1.613 to 1.631) overall, and was elevated in each separate category of mental illness. ARRs were highest for alcohol/ drug disorders, schizophrenia and affective psychoses (2.404, 1.834 and 1.798 , respectively). The results were not changed by location (metropolitan, rural or remote addresses). However, the $4 \%$ of MHCs with no fixed address had a very low ARR of visits to GPs (0.058; 95\% CI 0.057 to 0.060$)$.

Conclusion MHCs visit GPs substantially more often than nonMHCs, with the exception of those with no fixed address who seldom see a GP at all.

\section{P2-170 DIRECT ESTIMATION OF TOBACCO-ATTRIBUTABLE CANCER MORTALITY IN POLAND}

doi:10.1136/jech.2011.142976j.5

M Manczuk. ${ }^{*}$ Cancer Center and Institute of Oncology, Warsaw, Poland

Introduction The aim of the present study was to estimate the number cancer deaths, and their proportion over total deaths in that age ranges, attributable to tobacco in Poland.

Methods The calculation of tobacco-attributable mortality was based on the combination of RRs and prevalence of exposure. The selection of tobacco-related diseases and causes of death relied on recent comprehensive reviews by the International Agency for Research on Cancer [IARC, 2004] and the U.S. Government [USDHHS, 2004]. The set of RRs was derived from the Cancer Prevention Study II (CPS-II). Data on smoking prevalence come from national survey studies. Data on cancer mortality separately for 11 cancer sites related to tobacco were obtained form National Cancer Registry. To introduce into the model the latency effect demonstrated for most chronic health effects of tobacco, period of 20 years latency between exposure and death was implemented.

Results In 2005 in Poland there were 24222 cancer deaths among men (197.3/100000) and 5177 among women (35.8/100000) attributed to active tobacco smoking. Among eleven cancer sites attributed to tobacco, the biggest killer was lung cancer with 15478 deaths among men (60.4/100 000) and 3538 deaths among women $(10.7 / 100000)$

Conclusion Direct estimations (based on smoking prevalence) can be successfully used to calculate tobacco burden. In Poland in tobaccorelated cancers kill substantial number of men and women every year. Quantifying the size of tobacco burden gives arguments for evidence-based tobacco control intervention.

\section{P2-171 PAIN AMONG OLDER PEOPLE AND ITS IMPACT ON DISABILITY: A 10/66 CROSS-SECTIONAL POPULATION- BASED SURVEYS IN LATIN AMERICA, INDIA AND CHINA}

doi:10.1136/jech.2011.142976j.6

\footnotetext{
${ }^{1} \mathrm{P}$ Manzolli, ${ }^{2} \mathrm{D}$ Acosta, ${ }^{3} \mathrm{M}$ Guerra, ${ }^{4} \mathrm{Y}$ Huang, ${ }^{5} \mathrm{~K}$ S Jacob, ${ }^{6} \mathrm{~J}$ Rodriguez, ${ }^{7} \mathrm{~A}$ Salas, ${ }^{8} \mathrm{~A}$ L Sosa, ${ }^{9} \mathrm{~J}$ Williams, ${ }^{10} \mathrm{M}$ Prince, ${ }^{10} \mathrm{C}$ Ferri. ${ }^{1}$ Universidade Federal do Rio Grande do Sul, Porto Alegre, Brazil; ${ }^{2}$ Universidad Nacional Pedro Henriquez Ureña (UNPHU), Internal Medicine Department, Geriatric Section, Santo Domingo, Dominican Republic; ${ }^{3}$ Psychogeriatric Unit, National Institute of Mental Health "Honorio Delgado Hideyo Noguchi", Lima, Peru; ${ }^{4}$ Peking University, Institute of Mental Health, Beijing, China; ${ }^{5}$ Christian Medical College, Vellore, India; ${ }^{6}$ Facultad de Medicina Finlay-Albarran,
}

Medical University of Havana, Havana, Cuba; ${ }^{7}$ Medicine Department, Caracas University Hospital, Faculty of Medicine, Universidad Central de Venezuela, Caracas, Venezuela; ${ }^{8}$ National Institute of Neurology and Neurosurgery of Mexico, National University Autonomous of Mexico, Mexico City, Mexico; ${ }^{9}$ Department of Community Health, Voluntary Health Services, Chennai, India; ${ }^{10}$ Centre for Public Mental Health, Health Services \& Population Research Department, Institute of Psychiatry, King's College London, Health Services \& Population Research Department, London, UK

Introduction Pain is an important indicator of health mainly among older adults. The aim of the present study is to describe the prevalence of pain and to examine the relationship between pain and disability in elderly.

Methods One-phase cross-sectional surveys of all residents aged 65 years and over $(n=15177)$ living in eight low- and middle-income countries. Pain was measured using three questions, about frequency, severity and extent of limitation associated with pain in the previous 4 weeks. Disability was assessed using the 12 item WHODAS 2.0. We calculated the crude and standardised prevalence of pain and used Poisson regression prevalence ratios, to estimate the association between pain and severe disability, and to generate population attributable prevalence fractions (PAPF).

Results The overall prevalence of pain-defined as any type of pain in the last 4 weeks - ranged between 15.1\% (China) and 46.0\% (Peru) in urban sites and between $33.5 \%$ (Peru) and 58.8\% (India) in rural sites. Pain was associated with severe disability, prevalence ratio pooled estimate was 1.49 (95\% CI 1.21 to 1.78), adjusted for depression, number of impairments and chronic disease diagnoses. The adjusted PAPF showed that $39.2 \%$ of severe disability could be explained by pain.

Conclusion The results show that the prevalence of pain in the elderly is high. Pain seems to be an important contributor to disability among older people. Pain in older people is an increasingly important health issue worldwide, and one that requires urgent worldwide attention from the public health and clinical perspectives.

\section{P2-172 ADVERSE HEALTH EVENTS DURING OCCUPATIONAL EXPOSURE TO PESTICIDES, IN CORDOBA, ARGENTINA. AN ESTIMATION OF ITS IMPACT ON AGRICULTURAL APPLICATORS HEALTH}

doi:10.1136/jech.2011.142976j.7

${ }^{1} \mathrm{~B}$ Mariana, ${ }^{* 2} \mathrm{~F}$ Ricardo, ${ }^{1} \mathrm{~S} \mathrm{M}$ Ines, ${ }^{1} \mathrm{~L}$ M Josefina, ${ }^{1} \mathrm{~B}$ Marcelo, ${ }^{1} \mathrm{~B}$ Mariano, ${ }^{1} \mathrm{M}$ A Lia, ${ }^{1} \mathrm{D}$ M del Pilar. ${ }^{1}$ Universidad Nacional de Córdoba, Córdoba, Argentina; ${ }^{2}$ Universidad Católica de Córdoba, Córdoba, Argentina

The use of pesticides undeniably comes with some risks. It is understood that people who work with pesticides typically have much higher exposure than general public. The present work evaluated the prevalence of adverse events occurring during occupational exposure as an estimation of health impact. Also associations with several demographic characteristics, lifetime exposure years, working practices and protection level, considering a personal protection equipment (PPE) index, were investigated. Our results shown that the study population is relatively young $(34.9 \pm 11.04 \mathrm{y})$; $71 \%$ have up to 10 years of exposure and $80 \%$ are under 45 years of age, being $11.8 \%$ illiterate or with incomplete primary school. PPE is not adequate used in around $70 \%$ of the workers. Agrochemical prescription, indicated by an agricultural engineer, is only used by $38 \%$ of workers and the percentage of use of modern technologies (such as crop sprayers equipped with cabs and activated charcoal filter) is low. Forty four percent answered to have irritative symptoms (skin, eyes, nausea and vomiting) frequently, 35\% requiring medical consultation and $5.4 \%$ hospitalisation. Other symptoms such as headache, tiredness, nervousness or depression were also reported. The lifetime exposure is associated with irritative signs, 